\title{
Different tobacco cultivation regions lead to variations in tobacco leaf gene expression profiles involved in carbonhydrate metabolism and ion transportation
}

\author{
LiMing Lu, WenShan Xu, Yong Chen, ZongXiang Tang, Lei Liu and LiQin Li* \\ Agronomy College, Sichuan Agricultural University, Huimin Road 211\#, Wenjiang, Chengdu, Sichuan Province, \\ 611130, People's Republic of China
}

\author{
*Corresponding author: lilq88@126.com
}

\begin{abstract}
Tobacco is an important crop economically in China, and tobacco quality varies across its cultivated regions. To unveil the mechanisms that result in such varied tobacco leaf qualities, we have analyzed the tobacco leaf gene expression profiles of four areas in Sichuan province, China. Tobacco leaves in the $12^{\text {th }}$ position were collected from plants in four different regions, namely Huili, Miyi, Xingwen and Jiange, and submitted for microarray analysis. Gene expression levels from Miyi group were used as control, and that from Jiange, Xingwen and Huili were compared to Miyi group respectively. A total of 5154 differentially expressed genes (DEGs) were detected, among which 2731 genes were up-regulated and 2423 genes were down-regulated. Based on gene ontology (GO) analysis, significant DEGs were classified into 15 categories $(P \leq 0.05)$ based on properties pertaining to transferase activity, transmembrane transporter activity, binding, transcription regulator activity, metabolic processes, secondary metabolic processes, etc. Our results also show that these DEGs were significantly enriched in 11 pathways, including metabolic pathways, starch and sucrose metabolism, photosynthesis, etc. Our study suggests that many of the genes involved in various plant physiological and biochemical processes significantly differed across these four different regions, which may explain their differences in tobacco leaf quality.
\end{abstract}

Keywords: Nicotiana tabacum L, transcriptome, ecology, quality, gene chips.

Abbreviations: AGP_ADP glucose pyrophosphorylase; DEGs_differentially expressed genes; GER_germin-like protein; GO_gene ontology; HTA_Helanthus tuberosus agglutinin; NCED_9-cis-epoxycarotenoid dioxygenase; qPCR_quantitative reverse transcription polymerase chain reaction; RIP_ribosome inactivating protein.

\section{Introduction}

Tobacco is an important commercial crop in China. Because tobacco with high leaf quality can be sold at higher prices, farmers are more concerned with tobacco leaf quality than with yield. Leaf quality varies across tobacco cultivation areas and can be determined based on certain conventional chemical indicators, such as total sugar, total nitrogen, nicotine, starch and potassium content, etc (Lu et al., 2015). Among the many factors that can affect tobacco leaf quality, eco-climate is particularly important ( $\mathrm{Li}$ and Dai, 2013). Some studies (Shi et al., 1999; Yang et al., 2007; Huang et al., 2009; Yun et al., 2010; Shi et al., 2012) have revealed that light wave length and/or light intensity can significantly affect tobacco leaf dry-matter accumulation, aroma substance content, enzymatic activity, sugar content, etc. Although these individual studies unveil certain parts of the mechanisms related to the effects of eco-climate on tobacco leaf quality, the overall mechanism can only be understood by analyzing gene expression profiles across the entire genome. Recently, transcriptome analysis technology has been widely used to unveil the mechanisms involved in plant responses to abiotic stresses (Wasaki et al., 2003; Misson et al., 2005; Lian et al., 2006; Wasaki et al., 2006; Li et al., 2010; Krapp et al., 2011; Wang et al., 2011; Woo et al., 2012; Ma et al., 2012). This technology has also been used to investigate the mechanisms that determine different crop qualities. Long et al. (2011) have compared the gene expression profiles of flax obtained from the Hunan and Yunnan provinces in China and classified most of the differentially expressed genes detected under the functional category of cell structure.

In tobacco, gene expression profile analysis was also used to unveil the relationship between eco-climate and tobacco leaf aroma characteristics (Cui et al., 2011; Zhang et al., 2014). The result showed that differences in carbon metabolism, phenylpropanoid synthesis, alkaloid synthesis and terpenoid metabolism may account for the different tobacco leaf aroma characteristics of the Henan, Yunnan and Guizhou regions. However, tobacco leaf aroma characteristics is only one aspect of tobacco leaf quality. The relationship between conventional chemical components, which mainly determine tobacco leaf quality, and eco-climate at gene expression level remains unknown. Sichuan Province is an important tobacco cultivation region in China, and Miyi, Jiange, Xingwen and Huili are four typical tobacco cultivation areas in eco-climate in this province. Among them, tobacco leaf quality from Miyi is considered the best in the province. Lu et al. (2015) have measured the total sugar, total nitrogen, potassium and starch contents of leaves obtained from the four typical tobacco cultivation areas. The results showed that total sugar contents of leaves from Huili and Miyi were higher than those of the other two sites, while the starch contents of the former were lower than those from Xingwen and Jiange. Therefore, to uncover the relationship 
between tobacco conventional chemical components formation and eco-climate at gene expression level we collected tobacco leaves from these four ecological zones, and analyzed their gene expression profiles. Our results revealed that global changes in tobacco leaf gene expression regarding photosynthesis and ion transportation existed across the four areas.

\section{Results and Discussion}

\section{Tobacco gene expression profiles indicate global changes across tobacco cultivation areas}

We analyzed tobacco leaf gene expression profiles in leaves cultivated in Xingwen, Jiange, Huili and Miyi during the tobacco topping period and compared the strengths of gene expression signals in leaves collected from these regions. We considered 2-fold or greater changes in gene expression ratios to indicate up-regulation and 0.5 -fold or lower changes in gene expression ratios to indicate down-regulation. At topping period, compared to Miyi a total of 5154 differentially expressed genes were detected, among which 2731 genes were up regulated and 2423 were down regulated (Table 1). Among these 5154 DEGs, 359 genes were shared by tobacco leaves from the Xingwen, Jiange and Huili regions, including 192 up-regulated genes and 167 down-regulated genes.

To reveal the similarities and differences in genes expression profiles obtained for leaves collected from different tobacco cultivation regions, we performed a hierarchical cluster analysis. The results showed that genes expression patterns were similar among tobacco leaves from the Xingwen, Jiange and Huili regions and differed from the patterns observed in tobacco leaves obtained from the Miyi region (Fig. 1). To verify the microarray data obtained, we performed qPCR analysis of the 12 randomly selected genes and found that the expression patterns of 10 out of 12 genes agreed quite well with the corresponding microarray data (Fig. 2).

\section{Functional classification of differentially expressed genes}

We classified the functions of these DEGs by means of GO (Gene Ontology) analysis (http://www.geneontology.org) and found that these DEGs were significantly enriched with regard to 15 classifications $(P \leq 0.05)$, including transferase activity, binding, etc. (Fig. 3). These 15 classifications included genes with up- or down- regulated expression levels. The percentages of down-regulated genes showing enrichment with regard to these 15 classifications were higher than those obtained for up-regulated genes, except in the case of transcriptional activator activity. These results suggest that the transcriptional activator activity of tobacco leaves cultivated in Miyi was lower than that of the other three regions.

\section{Differential expression genes enriched metabolic pathway analysis}

Differentially expressed genes were significantly enriched in 11 pathways (Table 2), among which only the metabolic pathway simultaneously existed in all three comparison groups. Pathways existing in two comparison groups at the same time were endocytosis, thiamine metabolism, starch and sucrose metabolism, and photosynthesis. Because starch and sugar are tobacco leaf quality indicators, differences in the expression patterns of associated genes may indicate that primary metabolism, such as photosynthesis and starch and sucrose metabolism, and secondary metabolism such as carotenoid and flavonoid biosyntheses play key roles in determining tobacco output and quality.

\section{DEGs in photosynthesis}

Light reaction is a process involving light collection and energy-conversion and is, therefore, vital for plant photosynthesis (de Bianchi et al., 2011). The present study has identified six differentially expressed genes (DEGs) involved in the light reaction of photosynthesis (Table 3), all of which encode proteins involved in photosystem I or photosystem II. $L H C$ genes encode light-harvesting complex proteins; thus, $L H C$ expression levels may change under conditions of drought stress and/or according to light strength (Peremarti et al., 2014; Chen et al., 2010). The observed down-regulation of all six of these DEGs may indicate more efficient light collection and energy conversion in tobacco leaves from the Miyi region compared to the other three areas, which may account for the higher sugar content measured in leaves collected from the Miyi region (Lu et al, 2015).

Carbon fixation is an important biochemical reaction that is essential for photosynthetic sugar synthesis. Upon comparing the gene expression profiles for tobacco leaves obtained from the Huili and Miyi regions, we have identified three DEGs involved in the carbon fixation process (Table 4). Of these three genes, SBPase was up regulated, and $P P C 3$ and $B C A 3$ were down regulated. SBPase is vital to the Calvin circle in photosynthesis. Previous studies have shown that, in transgenic organisms, overexpression of $F B P / S B P$ ase genes enhances $\mathrm{CO}_{2}$ assimilation rates and increases biomass production (Miyagawa et al., 2001; Yabuta et al., 2008; Ichikawa et al., 2010; Ogawa et al., 2015). Higher expression of SBPase may explain why the sugar contents of tobacco leaves are higher in the Huili region compared to the Miyi region.

Because carbonic anhydrase (CA) plays a key role in the transformation of $\mathrm{CO}_{2}$ to $\mathrm{HCO}_{3}^{-}$, which can be utilized for carbon fixation, changes in $C A$ gene expression levels and carbonic anhydrase activity may result in changes in plant photosynthesis and, eventually, differences in plant development (Sun et al., 2001; He et al., 2012). Therefore, our results suggest that changes in the expression levels of genes involved in tobacco photosynthesis may lead to differences in tobacco photosynthesis and sugar contents across cultivation areas.

\section{DEGs in starch and sucrose metabolism pathway}

Transcriptome profiling reveals differences in starch and sucrose metabolism across the four regions considered herein. Because sugar has an important physiological role in providing energy as well as the carbon frames in fresh tobacco leaves, it may also greatly influence the smoke quality of tobacco leaves. To some extent, tobacco leaf quality improves with increased sugar content (Wang et al., 2013). Therefore, starch and sucrose contents are among the main indexes of tobacco leaf quality (Dong et al. 2015).

Previous studies showed that genes encoding starch biosynthesis were significantly changed under conditions of drought and heat stress, resulting in decreased starch contents (Yi et al., 2014; Cao et al., 2015). In the present study, we detected 14 DEGs involved in the starch and sucrose metabolism pathway (Table5). These genes are all related to starch and sucrose synthesis or degradation and are vital for plant development. Therefore, changes in their expression levels may indicate differences in starch and sucrose accumulation across different tobacco cultivation regions 
Table 1. Number of significantly differentially expressed genes (DEGs) between different cultivation regions.

\begin{tabular}{llllll}
\hline & Total & Up-regulated & Percent $(\%)$ & Down-regulated & Percent $(\%)$ \\
\hline Xingwen vs Miyi & 1633 & 837 & 51.26 & 796 & 48.74 \\
Jiange vs Miyi & 2122 & 1202 & 56.64 & 920 & 43.36 \\
Huili vs Miyi & 1399 & 692 & 49.46 & 707 & 50.54 \\
\hline
\end{tabular}

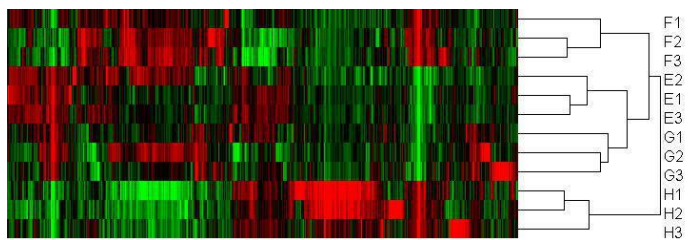

Fig 1. Cluster analysis of tobacco leaf gene expression profiles in four regions. E1-E3 refers to samples from Xingwen, F1-F3 refers to samples from Jiange, G1-G3 refers to samples from Huili and H1-H3 refers to samples from Miyi. Red indicates up-regulated genes and green indicates down-regulated genes.

Table 2. Differentially expressed genes enriched pathways.

\begin{tabular}{|c|c|c|c|c|c|c|}
\hline \multirow{2}{*}{ Pathway Name } & \multicolumn{2}{|c|}{ Xingwen vs Miyi } & \multicolumn{2}{|c|}{ Jiange vs Miyi } & \multicolumn{2}{|c|}{ Huili vs Miyi } \\
\hline & $P$-value & Percent & $P$-value & Percent & $P$-value & Percent \\
\hline Metabolic pathways & 0.006 & $6.60 \%$ & 0 & $9.79 \%$ & $8.00 \mathrm{E}-04$ & $6.21 \%$ \\
\hline Endocytosis & & & 0.0023 & $18.18 \%$ & 0.0099 & $12.12 \%$ \\
\hline Thiamine metabolism & 0.0096 & $42.86 \%$ & & & 0.0064 & $42.86 \%$ \\
\hline Plant-pathogen interaction & & & 0.0013 & $14.49 \%$ & & \\
\hline Amino sugar and nucleotide sugar metabolism & & & $5.00 \mathrm{E}-04$ & $17.98 \%$ & & \\
\hline Biosynthesis of phenylpropanoids & & & 0.0025 & $11.74 \%$ & & \\
\hline Starch and sucrose metabolism & 0.0094 & $11.32 \%$ & 0.0026 & $15.09 \%$ & & \\
\hline Flavonoid biosynthesis & & & 0.003 & $31.58 \%$ & & \\
\hline Phenylpropanoid biosynthesis & & & 0.0055 & $14.29 \%$ & & \\
\hline Glycerolipid metabolism & & & 0.0058 & $23.33 \%$ & & \\
\hline Photosynthesis - antenna proteins & & & & & 0 & $42.11 \%$ \\
\hline Photosynthesis & 0.0058 & $13.51 \%$ & & & $6.00 \mathrm{E}-04$ & $14.86 \%$ \\
\hline
\end{tabular}

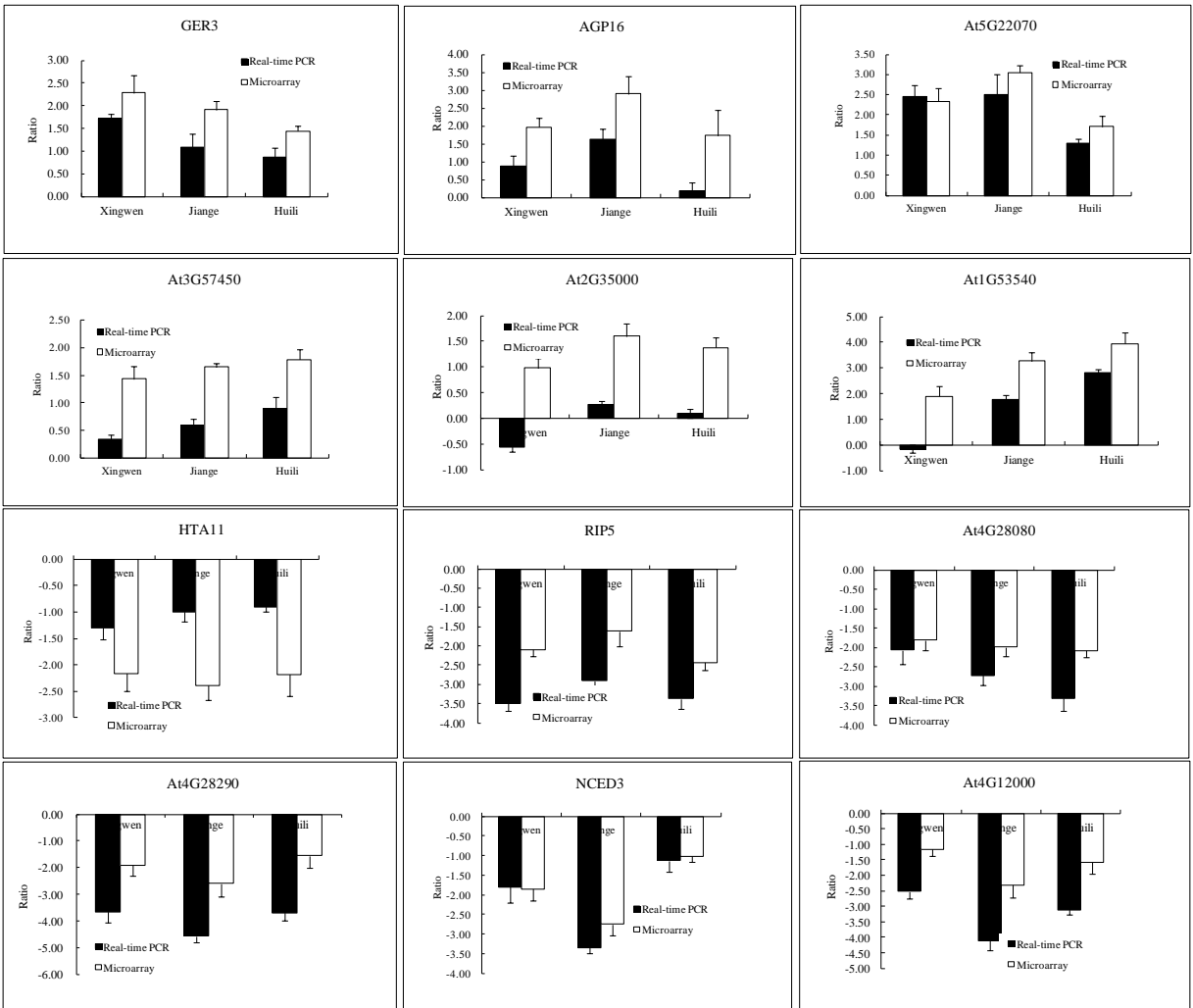

Fig 2. Verification of genes expression patterns by real-time PCR. Ratios refers to the comparison of selected gene expression levels at the regions indicated to Miyi. AGP16 refers to ADP glucose pyrophosphorylase 16, GER3 refers to germin-like protein3, HTA11 refers to Helanthustuberosus agglutinin 11, RIP 5 refers to ribosome inactivating protein 5, NCED3 refers to 9-cis-epoxycarotenoid dioxygenase 3. The black box indicates expression patterns detected by real-time PCR, and white box indicates expression patterns detected by microarray. 
Table3. The differently expressed genes of antenna protein.

\begin{tabular}{llllllll}
\hline \multirow{2}{*}{ Symbol } & \multicolumn{2}{l}{ Xingwen vs Miyi } & Jiange vs Miyi & \multicolumn{2}{l}{ Huili vs Miyi } & Description \\
\cline { 2 - 6 } & FC & $P$-value & FC & $P$-value & FC & $P$-value & \\
\hline CAB1 & -1.234 & 0.024 & & -2.225 & 0.016 & Chlorophyll a-b binding protein 1 \\
LHB1B2 & -1.694 & 0.004 & & -1.733 & 0.000 & $\begin{array}{l}\text { Light-harvesting complex II chlorophyll a/b binding } \\
\text { protein } 1\end{array}$ \\
LHCA2 & & & & -1.062 & 0.007 & Photosystem I light harvesting complex protein \\
LHCA4 & & & & -1.142 & 0.041 & Photosystem I light harvesting complex protein \\
LHCB3 & -1.019 & 0.011 & & -1.072 & 0.009 & Light-harvesting chlorophyll B-binding protein 3 \\
LHCB5 & & & & -1.383 & 0.015 & Light-harvesting chlorophyll B-binding protein 5 \\
\hline Note: FC, fold change; $P \leq 0.05$ & & & & &
\end{tabular}

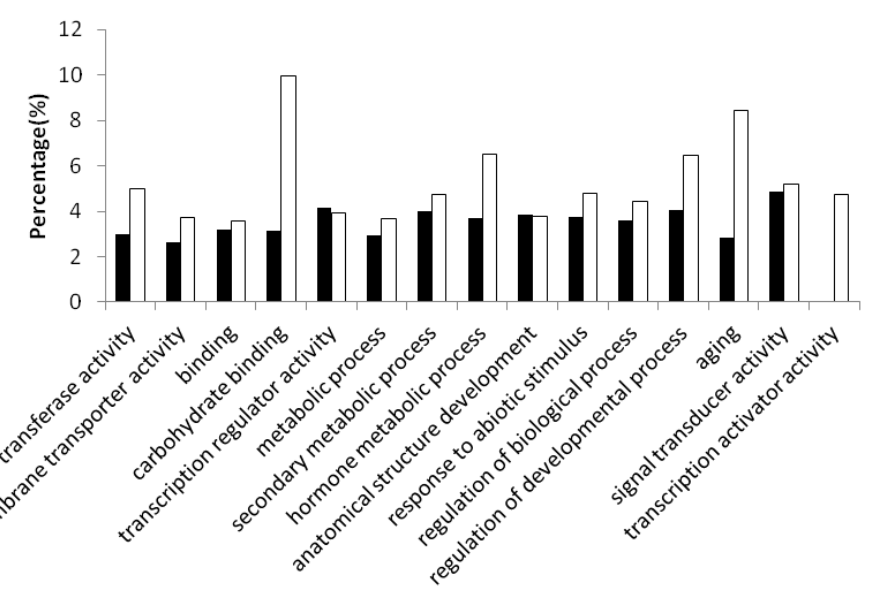

Fig 3. Functional classification of differentially expressed genes. Black box indicates the percentage of up-regulated genes and white box indicates that of down-regulated genes.

during the growth and development of flue-cured tobacco and may result in differences in tobacco leaf quality among the four regions investigated herein.

\section{DEGs involved in ion transportation across membranes}

Mineral elements are important for plant growth and development. Many of these elements, such as nitrogen, phosphorus, potassium, sulfur, calcium, magnesium and other trace elements, such as iron, manganese, zinc, boron, chlorine, molybdenum, are essential to tobacco quality and output formation. Some of these mineral elements are required in particular districts (Huang et al., 1999). In the present study, we successfully detected 24 DEGs involved in mineral transportation, of which six DEGs were related to potassium transportation (Table 6). The potassium content of tobacco leaf is significantly related to tobacco leaf quality (Wang and Ma, 2013; Hou et al., 2015; Tang et al., 2015). Lower expression levels of KUP3, KUP9 and SKOR in leaves from Jiange may result in lower tobacco leaf potassium content. Differences in these DEGs and resulting potassium differences may underlie the tobacco leaf quality differences observed among the four areas investigated herein. Compared to the Miyi region, the following DEGs were down regulated more than two-fold: CAX1, CAX3, ZIP1 and ZIP4. ZIP4 was the only gene shared by all three comparison groups. Zinc is also a tobacco quality-determining mineral element. According to Huang et al. (1999), zinc is particularly important with regard to tobacco leaf quality. In the present study, ZIP1 and ZIP4 were down-regulated compared to the Miyi region suggesting that down-regulation of these DEGs, particularly zinc transporters, may account for the lower leaf qualities observed for these three areas compared to the Miyi region.

\section{Materials and Methods}

\section{Plant materials and field experiments}

The "Yunyan 87" tobacco (Nicotiana tabacum L.) variety employed in this study was cultivated in the following four regions in Sichuan Province, China: Jiange, Xingwen, Miyi, and Huili. Miyi is located in the south of Sichuan Province. The distance from Jiange, Xingwen, and Huili to Miyi is 903 , 665 and 96.2 kilometers, respectively. Weather conditions of this four regions can be found in Table7.

In each of the four regions, a 600 -square-meter field was selected for study. Plots consisted of 16 rows with lengths of $30 \mathrm{~m}$, generally $120-\mathrm{cm}$ widths and $50 \mathrm{~cm}$ between tobacco plants. The sowing date (for tobacco seeds) and transplanting date (for tobacco seedlings) were January 18 and May 1, 2012, respectively. The farmers were responsible for field management practices, including sowing, transplanting, weeding and fertilization. Fertilizer containing $6 \mathrm{~kg} \mathrm{~N}, 8 \mathrm{~kg}$ $\mathrm{P}_{2} \mathrm{O}_{5}$ and $15 \mathrm{~kg} \mathrm{~K} \mathrm{~K}_{2} \mathrm{O}$ was manually applied to each plot after transplanting. Tobacco leaf samples were prepared using the middle leaves of the $12^{\text {th }}$ leaf position 70 days after transplanting. From each field, 30 tobacco plants were selected randomly, the leaves of which were cut and frozen in liquid nitrogen for RNA extraction.

\section{RNA isolation and hybridization to gene chips}

TRIzol reagent (Invitrogen, Carlsbad, USA) was used for total RNA extraction of tobacco leaf samples. A NanoDrop 2000 fluorospectrometer and formaldehyde denaturing gel electrophoresis were employed to assess the quality of the RNA extracted. An RNeasy Minikit (Qiagen, Valencia, USA) was used for total RNA purification, the product of which was used for cDNA and cRNA generation. An Agilent standard hybridization procedure was used for cRNA 
Table 4. The differently expressed genes of carbon utilization.

\begin{tabular}{lccl}
\hline Symbol & FC & $P$-value & Description \\
\hline BCA3 & -1.19 & 0.0061 & Beta carbonic anhydrase 3 \\
PPC3 & -1.04 & 0.0454 & Phosphoenolpyruvate carboxylase 3 \\
SBPase & 2.02 & 0.0001 & Sedoheptulose-1,7-bisphosphatase \\
\hline Note: FC, fold change; $P \leq 0.05$ & &
\end{tabular}

Note: FC, fold change; $P \leq 0.05$

Table 5. The differently expressed genes of starch and sucrose metabolism.

\begin{tabular}{|c|c|c|c|c|c|c|c|}
\hline \multirow{2}{*}{ Symbol } & \multicolumn{2}{|c|}{ Xingwen vs Miyi } & \multicolumn{2}{|c|}{ Jiange vs Miyi } & \multicolumn{2}{|c|}{ Huili vs Miyi } & \multirow{2}{*}{ Description } \\
\hline & $\mathrm{FC}$ & $P$-value & FC & $P$-value & FC & $P$-value & \\
\hline$A D G 1$ & 1.94 & 0.009 & 2.71 & 0.003 & & & Glucose-1-phosphate adenylyltransferase small subunit \\
\hline ATTPS7 & & & 1.22 & 0.017 & & & Putative alpha,alpha-trehalose-phosphate synthase \\
\hline BGLU12 & -1.14 & 0.042 & & & & & Beta glucosidase 12 \\
\hline$B G L U 15$ & & & -2.22 & 0.004 & & & Beta glucosidase 15 \\
\hline$D P E 2$ & -2.23 & 0.012 & -2.99 & 0.005 & -2.52 & 0.016 & 4-alpha-glucanotransferase \\
\hline GAUT12 & 2.97 & 0.001 & & & & & alpha-1,4-galacturonosyltransferase \\
\hline GAUT13 & 2.43 & 0.027 & 2.09 & 0.046 & & & Alpha-1,4-galacturonosyltransferase \\
\hline GAUT14 & & & 1.17 & 0.012 & & & Alpha-1,4-galacturonosyltransferase \\
\hline$H X K 1$ & -1.45 & 0.018 & -1.22 & 0.007 & & & Hexokinase 1 \\
\hline$P G M$ & -1.01 & 0.045 & -2.16 & 0.005 & -1.46 & 0.023 & Phosphoglucomutase \\
\hline PHS2 & -1.61 & 0.000 & -1.82 & 0.000 & -1.51 & 0.009 & Prostaglandin $\mathrm{H}$ synthase-2 \\
\hline SSI1 & & & & & -1.19 & 0.032 & Soluble starch synthase \\
\hline SUS3 & & & & & 1.01 & 0.004 & Sucrose synthase 3 \\
\hline TRE1 & 1.30 & 0.026 & & & & & Trehalase 1 \\
\hline
\end{tabular}

Table 6. The differently expressed genes of mineral elements.

\begin{tabular}{|c|c|c|c|c|c|c|c|}
\hline \multirow{2}{*}{ Symbol } & \multicolumn{2}{|c|}{ Xingwen vs Miyi } & \multicolumn{2}{|c|}{ Jiange vs Miyi } & \multicolumn{2}{|c|}{ Huili vs Miyi } & \multirow{2}{*}{ Description } \\
\hline & $\mathrm{FC}$ & $P$-value & FC & $P$-value & FC & $P$-value & \\
\hline AAT1 & & & -1.23 & 0.014 & & & Amino acid transporter 1 \\
\hline AMT2 & & & 1.25 & 0.006 & 1.07 & 0.049 & Ammonium transporter 2 \\
\hline NAXT1 & & & 1.10 & 0.034 & & & Nitrate excretion transporter 1 \\
\hline NRT1.7 & & & -1.00 & 0.020 & -1.26 & 0.013 & Nitrate transporter 1.7 \\
\hline PHT4;5 & & & -1.15 & 0.019 & & & Putative inorganic phosphate transporter $4 ; 5$ \\
\hline$H K T 1$ & 1.17 & 0.033 & 1.40 & 0.010 & & & Sodium transporter HKT1 \\
\hline KUP11 & -1.10 & 0.031 & & & & & Potassium transporter 11 \\
\hline KUP3 & & & 1.11 & 0.009 & 1.18 & 0.007 & Potassium transporter 3 \\
\hline KUP5 & 1.04 & 0.001 & & & & & Potassium transporter 5 \\
\hline KUP9 & -1.35 & 0.022 & -1.83 & 0.012 & & & $\mathrm{~K}+$ uptake permease 9 \\
\hline SKOR & & & -1.51 & 0.037 & & & Potassium channel SKOR \\
\hline CAX1 & -2.51 & 0.020 & & & & & Vacuolar cation/proton exchanger 1 \\
\hline$C A X 3$ & -2.53 & 0.015 & & & & & Vacuolar cation/proton exchanger 3 \\
\hline CAX5 & & & 1.38 & 0.047 & & & Vacuolar cation/proton exchanger 5 \\
\hline CAX9 & & & 1.32 & 0.040 & & & Cation/calcium exchanger 9 \\
\hline SULTR $2 ; 1$ & 1.16 & 0.006 & & & 1.68 & 0.007 & Sulfate transporter 2.1 \\
\hline SULTR1;1 & -1.69 & 0.006 & & & 1.14 & 0.001 & Sulfate transporter 1.1 \\
\hline ZIP4 & -2.09 & 0.021 & -2.08 & 0.009 & -1.61 & 0.024 & Zinc transporter 4 precursor \\
\hline$Z I P 1$ & -1.63 & 0.017 & & & -2.17 & 0.010 & Zinc transporter 1 \\
\hline ZIP6 & & & & & -1.10 & 0.026 & Zinc transporter 6 \\
\hline BOR4 & & & -1.59 & 0.007 & & & Boron transporter 4 \\
\hline$C L C-D$ & -1.221 & 0.002 & & & & & Chloride channel protein CLC-d \\
\hline$C C C 1$ & & & 1.29 & 0.025 & & & Cation-chloride co-transporter 1 \\
\hline MOT1 & & & -1.16 & 0.005 & & & Molybdate transporter 1 \\
\hline
\end{tabular}

Note: FC, fold change; $P \leq 0.05$

Table 7. Main weather indexes of the four regions.

\begin{tabular}{lllll}
\hline & Xingwen & Jiange & Huili & Miyi \\
\hline Annual average temperature $\left({ }^{\circ} \mathrm{C}\right)$ & 17.4 & 15.4 & 17 & 19.7 \\
Annual average rainfall $(\mathrm{mm})$ & 1234.7 & 1039.4 & 1000 & 1112.6 \\
Annual average humidity $(\%)$ & 66 & 69.1 & 69 & 65 \\
Annual sunshine $(\mathrm{hr})$ & 1010.3 & 1328.3 & 2400 & 2379.3 \\
\hline
\end{tabular}


labeling, fragmenting and hybridization to Agilent tobacco gene chips. The standard wash protocol was used, and a gene chip scanner (Agilent Microarray Scanner, Santa Clara, USA) was utilized for arrays scanning.

\section{Microarray data processing and analysis}

Feature Extraction software 10.7 and Gene Spring software 11.5.1 (Agilent Technologies, Santa Clara, USA) were used for raw data collection and normalization, respectively. A gene was selected for expression when it was detected in all three replicates and the $P$-value was less than 0.05 . Gene expression levels from Miyi group were used as control, and that from Jiange, Xingwen and Huili were compared to Miyi group respectively, forming three comparing group, Jiange $v s$ Miyi; Xingwen $v s$ Miyi and Huili $v s$ Miyi. The criteria for determining a differentially expressed gene (DEG) were that its expression level changed more than two-fold and that the corresponding $P$-value was less than 0.05 . Cluster 3.0 software

(http://bonsai.hgc.jp/ mdehoon/software/cluster/software.ht $\mathrm{m})$ was used for hierarchical cluster analysis, and differentially expressed genes (DEGs) were classified into functional categories using the gene ontology (GO) web service (http://www.geneontology.org).

\section{qRT-PCR analysis}

qRT-PCR analysis was used to confirm the reliability and repeatability of the microarray data. Toward this end, total RNA contents of tobacco leaf samples were extracted as described above, and DNaseI (RNase-free) (Takara, Dalian, China) was applied to eliminate the genomic DNA. Primer3 software (http://frodo.wi.mit.edu/primer3/input.htm) was used to design the gene specific primers utilized in this study. The gene used for normalisation of qRT-PCR data is Actin. The procedures for cDNA generation and qRT-PCR were conducted according to Ma et al. (2012).

\section{Conclusions}

In general agricultural practice, tobacco producers consider leaf quality more important than yield. Tobacco leaf qualities differ significantly based on the cultivation area. To understand the molecular-level mechanisms that determine tobacco leaf quality, we analyzed the gene expression profiles of tobacco leaves obtained from four tobacco cultivation areas and found that tobacco leaves collected from different planting areas exhibited significant variations in gene expression profiles. Up to 5154 differentially expressed genes (DEGs) were detected among the four areas. These DEGs were classified into 15 categories in relation to transferase activity, transmembrane transporter activity, etc., and were enriched in 11 pathways involved in primary and secondary metabolism, such as photosynthesis, starch and sucrose metabolism, carotenoid and flavonoid biosynthesis, etc. The results reported herein suggest that primary metabolism and secondary metabolism in the tobacco leaf play key roles in tobacco leaf quality.

\section{References}

Cao ZZ, Pang G, Wang FB, Wei KS, Li ZW, Shi CH, Geng W, Cheng FM (2015) Effect of high temperature on the expressions of genes encoding starch synthesis enzymes in developing rice endosperms. J Integr Agric. 14(4):642-659.
Chen WM, Zhang S, Fei BJ, Li W, Yu RQ, Xu H, Qiao DR, Cao Y (2010) Expression changes of lhcb and cao in Dunaliella salina under different light intensities. Chin J Appl Environ Biol. 2010,16(1): 18-22. (in Chinese with English abstract)

Cui H, Zhang ST, Yang HJ, Ji H, Wang XJ (2011) Gene expression profile analysis of tobacco leaf trichomes. BMC Plant Biol. 11:76-85.

de Bianchi S, Betterle N, Kouril R, Cazzaniga S, Boekema E, Bassi R, Dall'Osto L (2011) Arabidopsis mutants deleted in the light-harvesting protein Lhcb4 have a disrupted photosystem II macrostructure and are defective in photoprotection. Plant Cell. 23(7):2659-2679.

Dong WJ, Zhang ZF, Dou YQ, Zhao W, Duan SZ, Mao XX, Liu YJ, Chu XP (2015) Research on influencing factors of flue-cured tobacco starch content and its relationship with tobacco leaf quality. Guangdong Agric Sci. 42(8):11-16. (in Chinese with English abstract)

He X, Wang BY, Zhang XL, Ren LT, Kong GC (2012) Expression of carbonic anhydrase gene and enzyme activity in Triticale ( $X$ Triticosecale Wittmack) under salt stress. XinJiang Agric Sci. 49(7): 1197-1202. (in Chinese with English abstract)

Hou ZG, Yang HW, Yang YM, Chen YW (2015) Effects of potassium on the quality of flue-cured tobacco and the pathway for raising $\mathrm{K}$ content. $\mathrm{J}$ Anhui Agric Sci. 43(2):36-40. (in Chinese with English abstract)

Huang YJ, Fu Y, Dong ZJ, Deng HZ, Du XX (1999) Correlation between smoking quality and chemical elements, reducing sugar and nicotine contents in tobacco. Chin Tob Sci. 1999, 1:3-7. (in Chinese with English abstract)

Huang Y, Zhou JH, Zheng M, Yang HQ, Zhang NY (2009). Effect of UV-B on growth and development and secondary metabolism of flue-cured tobacco. Chin J Eco-Agric, 17:140-144 . (in Chinese with English abstract)

Ichikawa Y, Tamoi M, Sakuyama H, Maruta T, Ashida H, Yokota A, Shigeoka S (2010) Generation of transplastomic lettuce with enhanced growth and high yield. GM Crops. 1:322-326.

Li XQ, Dai F (2013) Recent advances on research of main ecological factors on tobacco quality. J Anhui Agric Sci. 41:531-532, 537. (in Chinese with English abstract)

Li L, Liu C, Lian X (2010) Gene expression profiles in rice roots under low phosphorus stress. Plant Mol Biol. 72(4-5):423-432.

Lian X, Wang S, Zhang J, Feng Q, Zhang LD, Fan DL, Li XH, Yuan DJ, Han B, Zhang QF (2006) Expression profiles of 10,422 genes at early stage of low nitrogen stress in rice assayed using a cDNA microarray. Plant Mol Biol. 60(5):617-631.

Long SH, Chen XB, Deng X, Qiu CS, Liu QN, Liu FH, Hao DM, Guo Y, Wang YF (2011) Analysis on gene expression profiles of flax under different environment using cDNA microarray. Plant Fib Sci China. 33(2): 65-69. (in Chinese with English abstract)

Lu LM, Ye KY, Tang ZX, Liu L, Chewn Y, Luo J, Huang YB (2015) Proteome analysis of tobacco leaves reveals dynamic changes in protein expression among different cultivation areas. Genet Mol Res, 14 (4): 15276-15284.

Krapp A, Brthome R, Orsel M, Mercey-Boutet S, Yu A, Castaings L, Elftieh S, Major H, Renou JP, DanielVedele F (2011) Arabidopsis roots and shoots show distinct temporal adaptation patterns toward nitrogen starvation[J]. Plant Physiol. 157(3):1255-1282. 
Ma TL,Wu WH, Wang Y (2012) Transcriptome analysis of rice root responses to potassium deficiency. BMC Plant Biol. 12: 161-173.

Misson J, Raghothama K G, Jain A, Jouhet J, Block MA, Bligny R, Ortet P, Creff A, Somerville S, Rolland N, Doumas P, Nacry P, Herrerra-Estrella L, Nussaume L, Thibaud MC (2005) A genome-wide transcriptional analysis using Arabidopsis thaliana affymetrix gene chips determined plant responses to phosphate deprivation. Proc Natl Acad Sci USA. 102(33): 11934-11939.

Miyagawa Y, Tamoi M, Shigeoka S (2001) Overexpression of a cyanobacterial fructose-1,6-/sedoheptulose-1,7-bisphosphatase in tobacco enhances photosynthesis and growth. Nat Biotechnol. 19:965-969.

Ogawa T, Tamoi M, Kimura A, Mine A, Sakuyama H, Yoshida E, Maruta T, Suzuki K, Ishikawa T, Shigeoka S (2015) Enhancement of photosynthetic capacity in Euglena gracilis by expression of cyanobacterial fructose-1,6-/sedoheptulose-1,7-bisphosphatase leads to increases in biomass and wax ester production. Biotechnol Biofuels. 8:80-90.

Peremarti A, Marè C, Aprile A, Roncaglia E, Cattivelli L, Villegas D, Royo C (2014) Transcriptomic and proteomic analyses of a pale-green durum wheat mutant shows variations in photosystem components and metabolic deficiencies under drought stress. BMC Genomics. 15:125-151.

Shi HZ., Han JH, Guan CY, Yuan T (1999) Effect of red and blue light proportion on leaf growth, carbon-nitrogen metabolism and quality in tobacco. Acta Agron Sin, 25:215-220. (in Chinese with English abstract)

Shi P, Shen GM, Xiang DA, Chen MH, Wang ZX, Xiang BK, Meng GX, Zhang L, Cao XH (2012). Relationships between main climatic factors and chemical components of flue-cured tobacco leaves in Enshi. Chin Tob Sci, 33:13-16. (in Chinese with English abstract)

Sun GC, Lin ZF, Lin GZ (2001) The carboxylation rate of rubisco and activity of carbonic anhydrase in plants from a subtropical forest grown at different light intensity. J Wuhan Bot Res. 19(4): 304-310 . (in Chinese with English abstract)

Tang XG, Feng XH, Qi F, Li ZZ, Li YY, Zhang R, Wang LB, $\mathrm{He}$ KX (2015) Effects of potassium and phosphorous fertilizers mixed application on yield and quality of flue-cured tobacco planted in red soil. Soil Fertil Chin. 4:76-81. (in Chinese with English abstract)

Wang D, Pan Y, Zhao X, Zhu L, Fu B, Li Z (2011) Genome-wide temporal-spatial gene expression profiling of drought responsiveness in rice. BMC Genomics. 12:149-163.
Wang LS, Ma R (2013) Major chemical components and quality and style and their relationships in full flavor tobacco growing regions. Chin Tob Sci. 34(5): 28-32. (in Chinese with English abstract)

Wasaki J, Yonetani R, Kuroda S, Shinano T, Yazaki J, Fujii F, Shimbo K, Yamamoto K, Sakata K, Sasaki T, Kishimoto N, Kikuchi S, Yamagishi M, Osaki M (2003) Transcriptomic analysis of metabolic changes by phosphorus stress in rice plant roots. Plant, Cell Environ. 26(9):1515-1523.

Wasaki J, Shinano T, Onishi K, Yonetant R, Sasaki T (2006) Transcriptomic analysis indicates putative metabolic changes caused by manipulation of phosphorus availability in rice leaves. J Exp Bot. 57(9):2049-2059.

Woo J, Macpherson C R, Liu J, Wang H, Kiba T, Hannah M A, Wang X J, Bajic V B, Chua N H (2012) The response and recovery of the Arabidopsis thaliana transcriptome to phosphate starvation. BMC Plant Biol. 12:62-83.

Yabuta Y, Tamoi M, Yamamoto K, Tomizawa K, Yokota A, Shigeoka S (2008) Molecular designing of photosynthesis-elevated chloroplasts for mass accumulation of a foreign protein. Plant Cell Physiol. 49:375-385.

Yang XY, Liu GS, Wu ZJ, Xia L, Xing XJ, Zhang JH, Du WM, Xie LW (2007). Effects of shading at different growth stages on the growth, development and quality of tobacco. Chin J Ecol, 26:1014-1020. (in Chinese with English abstract)

Yi B, Zhou YF, Gao MY, Zhang Z, Han Y, Yang GD, Xu WJ, Huang RD (2014) Effect of drought stress during flowering stage on starch accumulation and starch synthesis enzymes in sorghum grains. J Integrat Agric. 13(11):2399-2406.

Yun F, Liu GS, Shi HZ(2010). Interaction effects of light intensity and nitrogen supply on gas exchange, some enzyme activities in carbon-nitrogen metabolism and quality in flue-cured tobacco. Acta Agron Sin, 36:508-516. (in Chinese with English abstract)

Zhang ST, Yang YX, Yang HJ, Jia HF, Zhang HY, Cui H (2014) Analysis of differential gene expression profile for tobacco (Nicotiana tabacum L. $c v$.) leaf in three different ecological zones. J Agric. Biotechnol. 22(10):1251-1260. (in Chinese with English abstract) 